\title{
FC02-04
}

\section{GENETICS OF INTERMEDIATE PHENOTYPES IN SUICIDAL BEHAVOR - FOCUS ON AGGRESSION}

D. Rujescu ${ }^{1}$, A. Hartmann ${ }^{1}$, B. Schneider ${ }^{2}$, A. Thalmeier ${ }^{1}$, A. Schnabel ${ }^{2}$, K. Maurer $^{2}$, H. Moeller ${ }^{1}$, I. Giegling ${ }^{1}$

${ }^{1}$ Ludwig-Maximilian University, Munich, ${ }^{2}$ Goethe University, Frankfurt/Main, Germany

Family and twin studies point towards a partial heritability of suicidal behavior. We investigated the role of a comprehensive set of genes in this behavior. Their selection was driven by results from post mortem and genetic studies. 250 suicide attempters with various psychiatric disorders were compared with 2200 volunteers which were randomly selected from the general population. All subjects were administered standard psychiatric interviews including SCID as well as self-report questionnaires for anger-related traits. Especially, aggressive-impulsive behavior has been studied and associations with these intermediate phenotypes will be presented.

Additionally a large-scale gene expression analysis using cDNA-microarrays to identify new candidate-genes for suicide was conducted. We found several genes to be differentially expressed in the orbitofrontal cortex of suicide completers. Cross-validation experiments using quantitative RT-PCR validated a few genes so far. These genes have been genotyped in our patients and controls and associations with suicidal behavior and intermediate phenotypes, like aggression and impulsivity will be presented. 